From what precedes it follows that to each value of $r$ and $\alpha$ satisfying the conditions

$$
0<r<\rho, \quad \alpha=\text { any value }
$$

there corresponds one and but one pair of values $s, t$ satisfying the conditions

and the equations

$$
0<s<S_{1}, \quad t=\text { any value }
$$

$$
r \cos \alpha=\phi(s, t), \quad r \sin \alpha=\psi(s, t) .
$$

The implicit functions

$$
s=s(r, \alpha), \quad t=t(r, \alpha)
$$

so defined are of class $C^{\prime}$ in the neighborhood of any point $(r, \alpha)$ of the region (10), since by (8) the determinant $\Delta=\phi_{s} \psi_{t}-\phi_{t} \psi_{s}$ of equations (11) is always different from zero when $s$ and $t$ satisfy the inequalities (9). It is easily seen that any two values of $\alpha$ differing by a multiple of $2 \pi$ define with $r$ the same values of $s$ and $t$.

Princeton,

February, 1907.

\title{
SOME PARTICULAR SOLUTIONS IN THE PROBLEM OF $n$ BODIES.
}

BY DR. W. R. LONGLEY.

(Read before the American Mathematical Society, December 28, 1906.)

LET the masses of $n$ finite bodies be represented by $m_{1}, m_{2}, \ldots, m_{n}$. Suppose that the bodies lie always in the same plane, and that their coordinates with respect to their common center of mass as origin and a system of rectangular axes which rotate with the uniform angular velocity $N$ are, respectively, $\left(x_{1}, y_{1}\right),\left(x_{2}, y_{2}\right), \ldots,\left(x_{n}, y_{n}\right)$. Supposing that the bodies attract each other according to the newtonian law, the differential equations of motion are

$$
\begin{aligned}
& \frac{d^{2} x_{i}}{d t^{2}}-2 N \frac{d y_{i}}{d t}-N^{2} x_{i}=-\sum_{j=1}^{n} \frac{m_{j}\left(x_{i}-x_{j}\right)}{r_{i, j}^{3}}, \\
& \frac{d^{2} y_{i}}{d t^{2}}+2 N \frac{d x_{i}}{d t}-N^{2} y_{i}=-\sum_{j=1}^{n} \frac{m_{j}\left(y_{i}-y_{j}\right)}{r_{i, j}^{3}},
\end{aligned}
$$




$$
r_{i, j}=\sqrt{\left(x_{i}-x_{j}\right)^{2}+\left(y_{i}-y_{j}\right)^{2}} \quad\left(\begin{array}{l}
i=1,2, \cdots, n, \\
j \neq i
\end{array}\right) .
$$

If we assume that each body is revolving in a circle about the common center of mass of the system with the uniform angular velocity $N$, its coordinates with respect to the rotating axes are constants, and the derivatives of the coordinates with respect to the time are zero. Equations (1) therefore reduce to the following system of algebraic equations :

$$
\begin{aligned}
& -N^{2} x_{i}+\sum_{j=1}^{n} \frac{m_{j}\left(x_{i}-x_{j}\right)}{r_{i, j}^{3}}=0, \quad\left(\begin{array}{l}
i=1,2, \cdots, n, \\
j \neq i
\end{array}\right) . \\
& -N^{2} y_{i}+\sum_{j=1}^{n} \frac{m_{j}\left(y_{i}-y_{j}\right)}{r_{i, j}^{3}}=0
\end{aligned}
$$

It is to be observed that the equations

$$
\begin{aligned}
& m_{1} x_{1}+m_{2} x_{2}+\cdots+m_{n} x_{n}=0 \\
& m_{1} y_{1}+m_{2} y_{2}+\cdots+m_{n} y_{n}=0
\end{aligned}
$$

which express the fact that the origin of coordinates is at the center of mass, follow from the system (2), and may be used instead of two of equations (2).

This system of $2 n$ simultaneous algebraic equations involves the square of the angular velocity $N$, the $n-1$ ratios of the masses, and the $2 n-1$ ratios of the distances $x_{i}$ and $y_{i}$. Accordingly $n-1$ of these quantities may be chosen arbitrarily and, if the resulting equations are independent, the remaining $2 n$ quantities are determined by the relations (2). In order to admit physical interpretation, the quantity $N^{2}$ and the masses must be real and positive, while the coordinates must be real. With these restrictions it is not easy to discuss the general solutions of the system (2). Some interesting results may, however, be obtained by a study of special cases. If, in the problem of three bodies, the assumption is made that the triangle formed by the three bodies is isosceles, it may be shown that equations (2) can be satisfied only if the triangle is also equilateral, which is the well-known solution of Lagrange.

A particular case of the problem of four bodies will be con- 
sidered in detail. The system of equations to be satisfied is

$$
\begin{aligned}
& m_{1} x_{1}+m_{2} x_{2}+m_{3} x_{3}+m_{4} x_{4}=0,(a) \\
& -N^{2} x_{1}+\frac{m_{2}\left(x_{1}-x_{2}\right)}{r_{1,2}^{3}}+\frac{m_{3}\left(x_{1}-x_{3}\right)}{r_{1,3}^{3}}+\frac{m_{4}\left(x_{1}-x_{4}\right)}{r_{1,4}^{3}}=0,(b) \\
& -N^{2} x_{2}+\frac{m_{1}\left(x_{2}-x_{1}\right)}{r_{2,1}^{3}}+\frac{m_{3}\left(x_{2}-x_{3}\right)}{r_{2,3}^{3}}+\frac{m_{+}\left(x_{2}-x_{4}\right)}{r_{2,4}^{3}}=0,(c) \\
& -N^{2} x_{3}+\frac{m_{1}\left(x_{3}-x_{1}\right)}{r_{3,1}^{3}}+\frac{m_{2}\left(x_{3}-x_{2}\right)}{r_{3,2}^{3}}+\frac{m_{4}\left(x_{3}-x_{4}\right)}{r_{3,4}^{3}}=0,(d) \\
& m_{1} y_{1}+m_{2} y_{2}+m_{3} y_{3}+m_{4} y_{4}=0 \text {, } \\
& -N^{2} y_{1}+\frac{m_{2}\left(y_{1}-y_{2}\right)}{r_{1,2}^{3}}+\frac{m_{3}\left(y_{1}-y_{3}\right)}{r_{1,3}^{3}}+\frac{m_{4}\left(y_{1}-y_{4}\right)}{r_{1,4}^{3}}=0,(f) \\
& -N^{2} y_{2}+\frac{m_{1}\left(y_{2}-y_{1}\right)}{r_{2,1}^{3}}+\frac{m_{3}\left(y_{2}-y_{3}\right)}{r_{2,3}^{3}}+\frac{m_{4}\left(y_{2}-y_{4}\right)}{r_{2,4}^{3}}=0,(g) \\
& -N^{2} y_{4}+\frac{m_{1}\left(y_{4}-y_{1}\right)}{r_{4,1}^{3}}+\frac{m_{2}\left(y_{4}-y_{2}\right)}{r_{4,2}^{3}}+\frac{m_{3}\left(y_{4}-y_{3}\right)}{r_{4,3}^{3}}=0,(h)
\end{aligned}
$$

The assumption will be made that the bodies lie at the vertices of a parallelogram, and that opposite masses are equal,

$$
m_{1}=m_{3}=m, \quad m_{2}=m_{4}=M .
$$

Let the $x$-axis pass through $m_{1}$. Then the coordinates may be represented as follows:

$$
m_{1}(\alpha, 0), \quad m_{2}(\beta, \gamma), \quad m_{3}(-\alpha, 0), \quad m_{4}(-\beta,-\gamma) .
$$

Equations $(a)$ and $(e)$ are satisfied. Equation $(f)$ becomes $r_{1,2}^{3}=r_{1,4}^{3}$, whence $\beta=0$, and the parallelogram must be a rhombus. Putting $\beta=0$, equation (c) is satisfied. Equations $(b)$ and $(d)$ become identical, yielding

$$
N^{2}=\frac{2 M}{r_{1,2}^{3}}+\frac{2 m}{r_{1,3}^{3}},
$$

and equations $(g)$ and $(h)$ become identical, yielding

$$
N^{2}=\frac{2 m}{r_{1,2}^{3}}+\frac{2 M}{r_{2,4}^{3}} \text {. }
$$


The two values of $N^{2}$ thus found must be the same. Therefore the following relation determines the ratio of the masses when the ratio of the distances is given :

$$
\frac{M}{m}=\frac{r_{2,4}^{3}\left(r_{1,3}^{3}-r_{1,2}^{3}\right)}{r_{1,3}^{3}\left(r_{2,4}^{3}-r_{1,2}^{3}\right)}=\frac{\gamma^{3}\left\{8 \alpha^{3}-\left(\sqrt{\alpha^{2}+\gamma^{2}}\right)^{3}\right\}}{\alpha^{3}\left\{8 \gamma^{3}-\left(\sqrt{\alpha^{2}+\gamma^{2}}\right)^{3}\right\}} .
$$

The choice of the ratio of the distances is limited by the condition that the resulting ratio of the masses must be positive. This condition is expressed by the inequality

$$
\frac{1}{\sqrt{ } 3}<\frac{\gamma}{\alpha}<\sqrt{ } 3 .
$$

Hence, in the problem of four bodies revolving in circles about their common center of mass with the same uniform angular velocity, the rhombus is a possible configuration provided the following conditions are fulfilled : (1) Opposite masses are equal, (2) the ratio of the diagonals shall be greater than $1 / \sqrt{ } 3$ and less than $\sqrt{ } 3$. The ratio of the masses is then uniquely determined by equation (7), and the angular velocity by equation (5) or equation (6).

Suppose a fifth body is introduced at the center of the rhombus. What will be the effect upon the limits within which one may choose the ratio of the diagonals? The method of treatment is analogous to that which precedes. The number of equations to be satisfied is ten, which, under the assumption that the configuration is a rhombus with opposite masses equal, reduce to the following two :

$$
\begin{aligned}
& N^{2}=\frac{2 M}{\left(\sqrt{\alpha^{2}+\gamma^{2}}\right)^{3}}+\frac{2 m}{8 \alpha^{3}}+\frac{m_{5}}{\alpha^{3}}, \\
& N^{2}=\frac{2 m}{\left(\sqrt{\alpha^{2}+\gamma^{2}}\right)^{3}}+\frac{2 M}{8 \gamma^{3}}+\frac{m_{5}}{\gamma^{3}} .
\end{aligned}
$$

Taking $m_{5}$ as the unit of mass, there results the following condition upon the masses $m$ and $M$ and the ratio $\gamma / \alpha$ of the diagonals of the rhombus :

$$
M=\left\{\frac{\gamma^{3}\left\{8 \alpha^{3}-\left(\sqrt{\alpha^{2}+\gamma^{2}}\right)^{3}\right\}}{\alpha^{3}\left\{8 \gamma^{3}-\left(\sqrt{\alpha^{2}+\gamma^{2}}\right)^{3}\right\}}\right\} m+\frac{4\left(\alpha^{3}-\gamma^{3}\right)\left(\sqrt{\alpha^{2}+\gamma^{2}}\right)^{3}}{\alpha^{3}\left\{8 \gamma^{3}-\left(\sqrt{\alpha^{2}+\gamma^{2}}\right)^{3}\right\}} .
$$

Regarding $\gamma / \alpha$ as a parameter, this equation represents a straight line in the $m M$-plane. Only those pairs of values 
$(m, M)$ are admissible which represent a point in the first quadrant. Hence $\gamma / \alpha$ must be selected so that some portion of the straight line lies in the first quadrant. This condition will certainly be satisfied if the slope is positive, that is, if the coefficient of $m$ is positive. This condition is

$$
\frac{1}{\sqrt{ } 3}<\frac{\gamma}{\alpha}<\sqrt{ } 3,
$$

which is the same as the one found in the case of four bodies. If, in the case of four bodies, the ratio $\gamma / \alpha=1 / \sqrt{ } 3$ (or $\sqrt{ } 3$ ) be selected the ratio $(M / m)$ of the masses is infinite (or zero). If, in the case of five bodies, the ratio $\gamma / \alpha=1 / \sqrt{ } 3$ (or $\sqrt{3}$ ) be selected there results $m=-4$ (or $M=-4$ ), which are not admissible. If the slope of the line is negative, it may still lie partly in the first quadrant if the intercept on the $M$-axis is positive. It is easily verified, however, that values of the ratio $\gamma / \alpha$ which render the slope negative make the intercept negative. Hence the limits of the ratio of the diagonals are the same in the case of five bodies as in the case of four. In the latter case the ratio of the masses is uniquely determined by the choice of the ratio of the diagonals. In the case of five bodies there is a single infinitude of possibilities (except for $\gamma / \alpha=1, i . e$. , when the rhombus becomes a square).

The rhombus, which is a special case of the parallelogram, is also a special case of the contraparallelogram.* It is interesting to notice, in the general case, that there exists a condition on the distances independent of the masses. The contraparallelogram is obtained by making $r_{1,2}=r_{1,4}, r_{3,2}=r_{3,4}, m_{2}=m_{4}$. Under these assumptions the coordinates may be represented as follows : $m_{1}(\alpha, 0), m_{2}(\beta, \gamma), m_{3}(-\delta, 0), m_{4}(\beta,-\gamma)$. The equations of condition (4) reduce to the following set :

$$
\begin{aligned}
& m_{1} \alpha+2 m_{2} \beta-m_{3} \delta=0, \\
& N^{2} \alpha=\frac{2 m_{2}(\alpha-\beta)}{r_{1,2}^{3}}+\frac{m_{3}(\alpha+\delta)}{r_{1,3}^{3}}, \\
& N^{2} \beta=\frac{m_{1}(\beta-\alpha)}{r_{1,2}^{3}}+\frac{m_{3}(\beta+\delta)}{r_{2,3}^{3}}, \\
& N^{2} \gamma=\frac{m_{1}}{r_{1,2}^{3}}+\frac{2 m_{2}}{r_{2,4}^{3}}+\frac{m_{3}}{r_{2,3}^{3}} .
\end{aligned}
$$

* Cf. Andoyer, "Sur l'équilibre relatif de $n$ corps," Bull. astron., vol. 23, pp. 50-59 (Feb., 1906). 
Eliminating $N^{2}$ between the second and third of equations (10) there results the following condition upon the distances:

$$
\frac{\delta(\alpha-\beta)}{r_{1,2}^{3}}+\frac{\beta(\alpha+\delta)}{r_{1,3}^{3}}-\frac{\alpha(\beta+\delta)}{r_{2,3}^{3}}=0 .
$$

If equation (11) is satisfied, equations (10) furnish a system of two linear equations for the determination of the ratios of the masses. Hence in any case in which the solution is possible the determination of the masses is unique. If the conditions upon the distances can be satisfied, the fourth of equations (10) insures a real angular velocity.

Symmetric Configurations. In the case of three bodies at the vertices of an equilateral triangle there is no restriction upon the masses; they may be equal. In the case of four bodies at the vertices of a square the masses must be equal. In the case of $n$ bodies it is known that, if all the bodies are equal, the regular polygon of $n$ vertices is a possible configuration. It is known * also that if $n$ equal masses are arranged at the vertices of a regular polygon, $n$ other equal masses may be arranged at the vertices of another regular polygon, such that every mass of the second set lies upon a bisector of a central angle of the first polygon; that is, the $2 n$ masses are arranged upon the circumferences of two concentric circles. The configuration is symmetric with respect to the line joining any one of the bodies to the center and the masses are distributed symmetrically with respect to this line. A configuration possessing these properties may be called a symmetric configuration.

For the consideration of symmetric configurations it is convenient to employ polar coordinates. The equations of condition, analogous to the system (2), are

$$
\begin{aligned}
& -N^{2} r_{i}+\sum_{j=1}^{n} \frac{m_{j}\left\{r_{i}-r_{j} \cos \left(\theta_{i}-\theta_{j}\right)\right\}}{r_{i, j}^{3}}=0, \\
& \quad \sum_{j=1}^{n} \frac{m_{j} r_{j} \sin \left(\theta_{i}-\theta_{j}\right)}{r_{i, j}^{3}}=0 \quad\left(\begin{array}{c}
i=1,2, \cdots, n, \\
j \neq i
\end{array}\right) .
\end{aligned}
$$

It is obvious that for any symmetric configuration the equa-

* Cf. R. Hoppe, "Erweiterung der bekannten Speciallösung des Dreikörperproblems." Archiv der Math. und Phys., vol. 64, pp. 218-23. 
tions (13), involving $\sin \left(\theta_{i}-\theta_{j}\right)$, are satisfied. Some of the equations involving $\cos \left(\theta_{i}-\theta_{j}\right)$ become identical, and the number of independent equations to be satisfied is consequently reduced. Suppose the number of masses is $n=k l$, arranged on $l$ concentric circles; that is, upon each circle are $k$ equal masses. As $i$ takes the values $1,2, \ldots, k$, corresponding to the bodies on the circle of radius $r_{1}$, the resulting equations are identical ; as $i$ takes values corresponding to the $k$ masses on the circle of radius $r_{2}$, the equations are identical ; etc. Therefore the system (12) reduces to $l$ independent equations involving $N^{2}$, the $l-1$ ratios of the distances, and the $l-1$ ratios of the masses. When $N^{2}$ is eliminated from this system of $l$ equations there results a system of $l-1$ equations, linear and homogeneous in the masses, the coefficients being functions of the radii. The distances must be chosen so that the masses are positive. If the necessary conditions upon the distances can be satisfied, the ratios of the masses are uniquely determined by the solution of a linear system of equations.

As a first example suppose $n=8$. Four of the masses $\left(m_{1}=m_{2}=m_{3}=m_{4}=m\right)$ are arranged on a circle of radius $r$, and four $\left(m_{5}=m_{6}=m_{7}=m_{8}=M\right)$ upon a circle of radius $R$. The configuration may be made symmetric in two ways,

1) $\theta_{1}=\theta_{5}=0, \quad \theta_{2}=\theta_{6}=\frac{\pi}{2}, \quad \theta_{3}=\theta_{7}=\pi, \quad \theta_{4}=\theta_{8}=\frac{3 \pi}{2}$.

2)

$$
\begin{array}{llll}
\theta_{1}=0, & \theta_{2}=\frac{\pi}{2}, & \theta_{3}=\pi, & \theta_{4}=\frac{3 \pi}{2}, \\
\theta_{5}=\frac{\pi}{4}, & \theta_{6}=\frac{3 \pi}{4}, & \theta_{7}=\frac{5 \pi}{4}, & \theta_{8}=\frac{7 \pi}{4} .
\end{array}
$$

1) (Fig. 1). The equations of condition (12) reduce to the following set:

$$
\begin{aligned}
N^{2} r & =\frac{2 m r}{(\sqrt{2} r)^{3}}+\frac{2 m r}{(2 r)^{3}}+\frac{M(r-R)}{(R-r)^{3}}+\frac{2 M r}{\left(\sqrt{r^{2}+R^{2}}\right)^{3}}+\frac{M(r+R)}{(r+R)^{3}}, \\
N^{2} R & =\frac{m(R-r)}{(R-r)^{3}}+\frac{2 m R}{\left(\sqrt{r^{2}+R^{2}}\right)^{3}}+\frac{m(R+r)}{(R+r)^{3}}+\frac{2 M R}{(\sqrt{2} R)^{3}}+\frac{2 M R}{(2 R)^{3}}
\end{aligned}
$$

Selecting $r$ for the unit of distance and $m$ for the unit of mass, there results the following equation for the determination 
of $M$ when $R$ is given :

$$
\begin{gathered}
M\left\{\frac{1}{\sqrt{2} R^{3}}+\frac{1}{4 R^{3}}+\frac{1}{(R-1)^{2}}-\frac{2}{\left(\sqrt{1+R^{2}}\right)^{3}}-\frac{1}{(1+R)^{2}}\right\} \\
=\frac{1}{\sqrt{2}}+\frac{1}{4}-\frac{1}{R(R-1)^{2}}-\frac{2}{\left(\sqrt{1+R^{2}}\right)^{3}}-\frac{1}{R(1+R)^{2}} .
\end{gathered}
$$

If $R=2, M=0.2636$; if $R=5, M=9.5914$; if $R=10$, $M=308.814$.

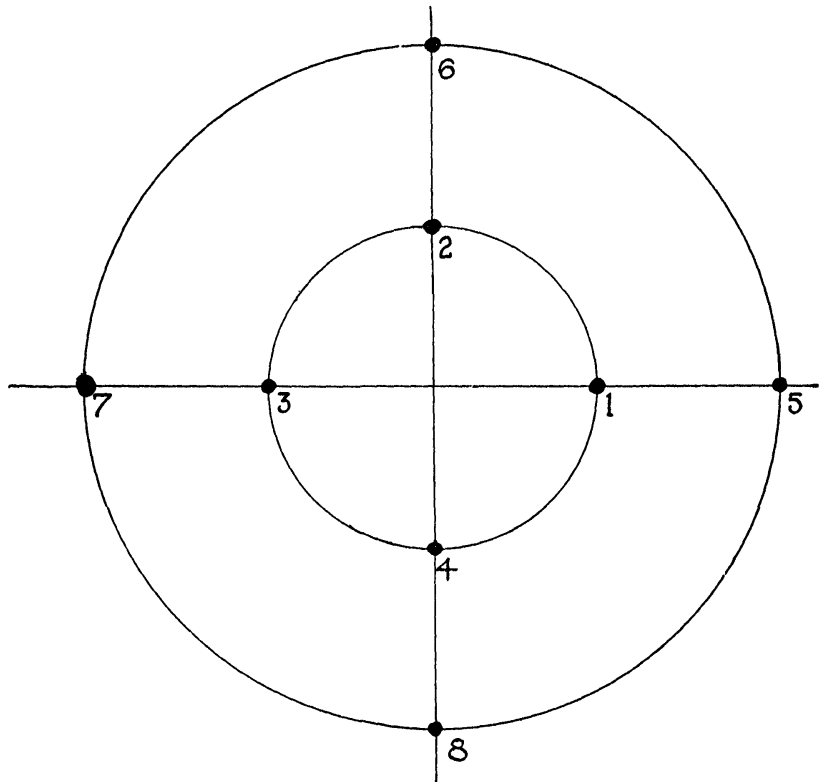

FIG. 1.

2) (Fig. 2). The equations of condition (12) reduce to the following set :

$$
\begin{aligned}
& N^{2} r=\frac{2 m r}{(\sqrt{2} r)^{3}}+\frac{2 m r}{(2 r)^{3}}+\frac{2 M\left(r-\frac{R}{\sqrt{2}}\right)}{\left(\sqrt{\left.r^{2}+R^{2}-\sqrt{2} r R\right)^{3}}\right.}+\frac{2 M\left(r+\frac{R}{\sqrt{2}}\right)}{\left(\sqrt{\left.r^{2}+R^{2}+\sqrt{2} r R\right)^{3}}\right.}, \\
& (16) \quad \\
& N^{2} R=\frac{2 m\left(R-\frac{r}{\sqrt{2}}\right)}{\left(\sqrt{r^{2}+R^{2}-\sqrt{2} r R}\right)^{3}}+\frac{2 m\left(R+\frac{r}{\sqrt{2}}\right)}{\left(\sqrt{\left.r^{2}+R^{2}+\sqrt{2} r R\right)^{3}}\right.}+\frac{2 M R}{(\sqrt{2} R)^{3}}+\frac{2 M R}{(2 \bar{R})^{3}} .
\end{aligned}
$$




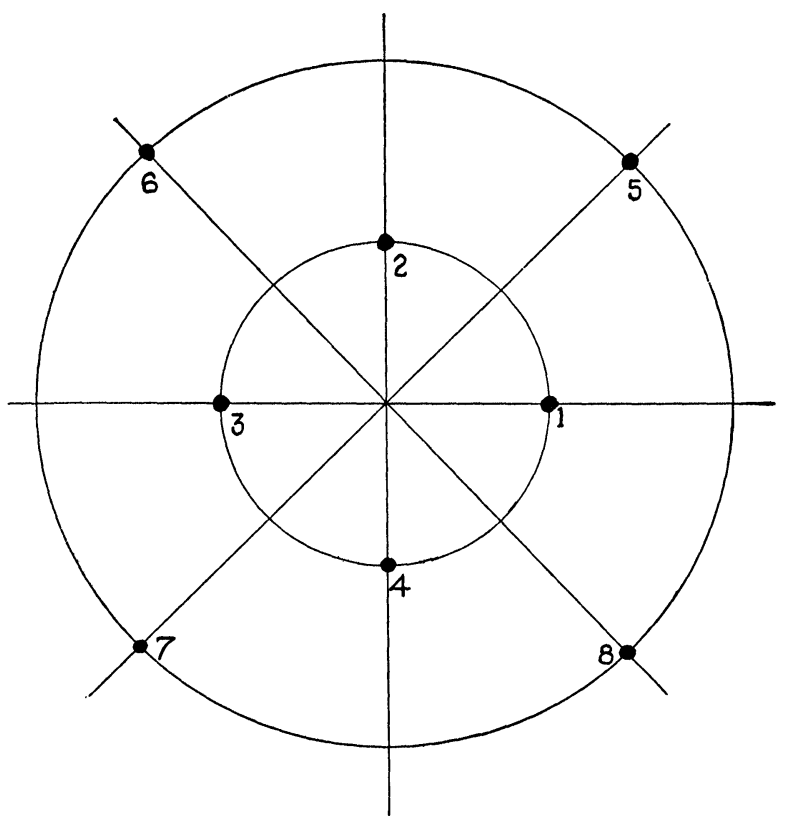

Fig. 2.

Selecting $r$ for the unit of distance and $m$ for the unit of mass, there results the following equation for the determination of $M$ when $R$ is given :

$$
\begin{gathered}
M\left\{\frac{1}{\sqrt{2} R^{3}}+\frac{1}{4 R^{3}}+\frac{R \sqrt{2}-2}{\left(\sqrt{1+R^{2}-R \sqrt{2}}\right)^{3}}-\frac{R \sqrt{2}+2}{\left(\sqrt{1+R^{2}+R \sqrt{2}}\right)^{3}}\right\} \\
\quad=\frac{1}{\sqrt{2}}+\frac{1}{4}-\frac{2 R-\sqrt{2}}{R\left(\sqrt{1+R^{2}-R} \sqrt{\overline{2}}\right)^{3}}-\frac{2 R+\sqrt{2}}{R\left(\sqrt{1+R^{2}+R \sqrt{2}}\right)^{3}} .
\end{gathered}
$$

If $R=2, M=2.622$; if $R=5, M=10.197$; if $R=10$, $M=330.37$.

As a second example suppose $n=8$ and that the masses are arranged on three circles as indicated in Fig. 3. Suppose

$$
\begin{gathered}
m_{1}=m_{2}=m, \quad m_{3}=m_{4}=M, \quad m_{5}=m_{6}=m_{7}=m_{8}=M^{\prime}, \\
r_{1}=r_{2}=r, \quad r_{3}=r_{4}=R, \quad r_{5}=r_{6}=r_{7}=r_{8}=R^{\prime} .
\end{gathered}
$$


The equations of condition (12) reduce to the following set:

$$
\begin{aligned}
N^{2} r=\frac{2 m r}{(2 r)^{3}}+\frac{2 M r}{\left(\sqrt{\left.r^{2}+R^{2}\right)^{3}}\right.}+\frac{2 M^{\prime} r}{\left(\sqrt{\left.r^{2}+R^{\prime 2}\right)^{3}}\right.} \\
+\frac{M^{\prime}\left(r-R^{\prime}\right)}{\left(R^{\prime}-r\right)^{3}}+\frac{M^{\prime}\left(r+R^{\prime}\right)}{\left(R^{\prime}+r\right)^{3}},
\end{aligned}
$$

$$
N^{2} R=\frac{2 m R}{\left(\sqrt{\left.r^{2}+R^{2}\right)^{3}}\right.}+\frac{M 2 R}{(2 R)^{3}}+\frac{2 M^{\prime} R}{\left(\sqrt{\left.R^{2}+R^{\prime 2}\right)^{3}}\right.}
$$

$$
\begin{array}{r}
+\frac{M^{\prime}\left(R-R^{\prime}\right)}{\left(R^{\prime}-R\right)^{3}}+\frac{M^{\prime}\left(R+R^{\prime}\right)}{\left(R+R^{\prime}\right)^{3}}, \\
N^{2} R^{\prime}=\frac{m\left(R^{\prime}-r\right)}{\left(R^{\prime}-r\right)^{3}}+\frac{m\left(R^{\prime}+r\right)}{\left(R^{\prime}+r\right)^{3}}+\frac{M^{\prime}\left(2 R^{\prime}\right)}{\left(2 R^{\prime}\right)^{3}}
\end{array}
$$$$
+\frac{2 M R^{\prime}}{\left(\sqrt{\left.R^{2}+R^{2}\right)^{3}}\right.}+\frac{2 M^{\prime} R^{\prime}}{\left(\sqrt{2} R^{\prime}\right)^{3}} .
$$

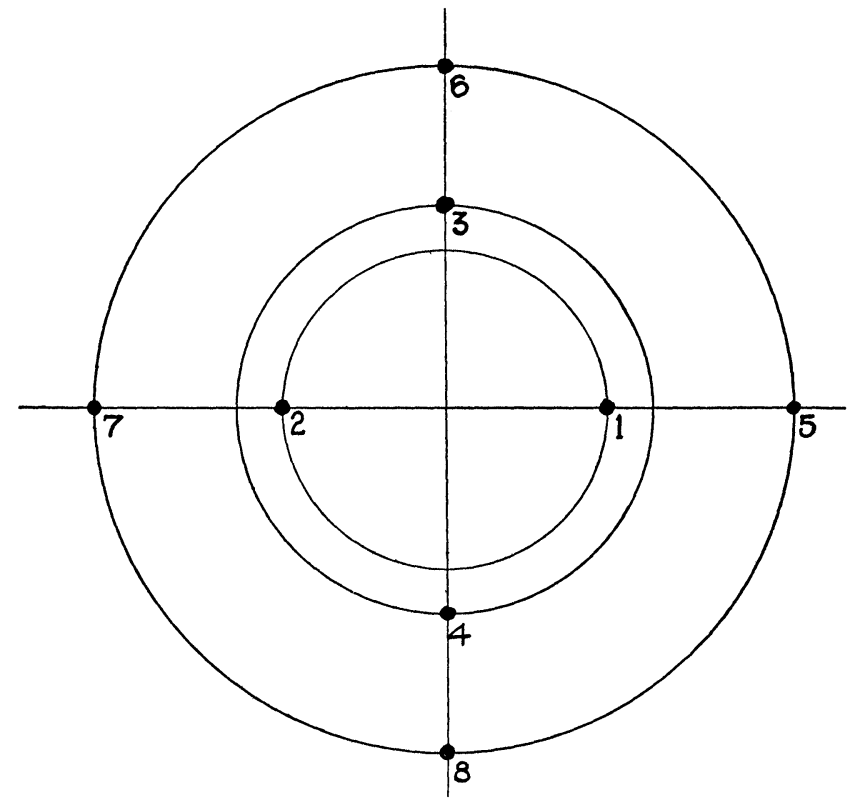

FIG. 3. 
Putting $r=1, m=1$, the following system of equations determines $M$ and $M^{\prime}$ when $R$ and $R^{\prime}$ are given :

$$
a M+b M^{\prime}=c, \quad a_{1} M+b_{1} M^{\prime}=c_{1},
$$

where

$$
\begin{aligned}
a= & \frac{2}{\left(\sqrt{\left.1+R^{2}\right)^{3}}\right.}-\frac{1}{4 R^{3}} \\
b= & \frac{2}{\left(\sqrt{\left.1+R^{\prime 2}\right)^{3}}-\frac{1}{\left(R^{\prime}-1\right)^{2}}+\frac{1}{\left(R^{\prime}+1\right)^{2}}\right.} \\
& \quad-\frac{2}{\left(\sqrt{\left.R^{2}+R^{\prime 2}\right)^{3}}\right.}+\frac{1}{R\left(R^{\prime}-R\right)^{2}}-\frac{1}{R\left(R^{\prime}+R\right)^{2}},
\end{aligned}
$$

$$
c=\frac{2}{\left(\sqrt{\left.1+R^{2}\right)^{3}}\right.}-\frac{1}{4}
$$$$
a_{\mathrm{r}}=\frac{2}{\left(V^{\prime} 1+R^{2}\right)^{3}}-\frac{2}{\left(\sqrt{\left.R^{2}+R^{\prime 2}\right)^{3}}\right.},
$$$$
b_{1}=\frac{2}{\left(\sqrt{\left.1+R^{\prime 2}\right)^{3}}\right.}-\frac{1}{\left(R^{\prime}-1\right)^{2}}+\frac{1}{\left(R^{\prime}+1\right)^{2}}-\frac{1}{4 R^{\prime 3}}-\frac{1}{\sqrt{2} R^{3}},
$$$$
c_{1}=\frac{1}{R^{\prime}\left(R^{\prime}-1\right)^{2}}+\frac{1}{R^{\prime}\left(R^{\prime}+1\right)^{2}}-\frac{1}{4} \text {. }
$$

If $\quad R=1.2, \quad R^{\prime}=5 ; \quad M=0.6331, \quad M^{\prime}=20.332$.

If $\quad R=1.3, \quad R^{\prime}=5 ; \quad M=0.4768, \quad M^{\prime}=16.081$.

If $\quad R=1.4, \quad R^{\prime}=5 ; \quad M=0.3140, \quad M^{\prime}=12.848$.

If $R=1.5, R^{\prime}=5$, the resulting value of $M$ is negative. In this case, also, the ratio of the diagonals of the rhombus formed by the inner bodies is limited, the upper limit lying between 1.4 and 1.5 .

The preceding discussion refers to configurations which are fixed in size, so that each body revolves about the center of mass of the system in a circle. The results can be extended at once to the case where the configuration preserves always the same shape, but varies in size so that each body describes an 
ellipse with the center of mass at one focus. As an example, consider the case of four bodies. The differential equations of motion in polar coördinates are

$$
\begin{gathered}
\frac{d^{2} r_{i}}{d t^{2}}-r_{i}\left(\frac{d \theta_{i}}{d t}\right)^{2}=-\sum_{j=1}^{4} \frac{m_{j}\left\{r_{i}-r_{j} \cos \left(\theta_{i}-\theta_{j}\right)\right\}}{r_{i, j}^{3}}, \\
r_{i} \frac{d^{2} \theta_{i}}{d t^{2}}+2 \frac{d r_{i}}{d t} \frac{d \theta_{i}}{d t}=-\sum_{j=1}^{4} \frac{m_{j} r_{j} \sin \left(\theta_{i}-\theta_{j}\right)}{r_{i, j}^{3}} \\
(i=1,2,3,4 ; j \neq i) .
\end{gathered}
$$

Suppose the bodies lie at the vertices of a rhombus, which may vary in size, but in which the ratio $\rho$ of the diagonals is constant, and selected within the limits $1 / \sqrt{ } 3<\rho<\sqrt{ } 3$. Suppose also that opposite masses are equal, $m_{1}=m_{3}=m$; $m_{2}=m_{4}=M$. Suppose that the ratio of the masses is determined by the relation $(7)[\gamma / \alpha=\rho]$. We may make the followlowing conditions upon the coordinates:

$$
\begin{gathered}
\theta_{1}=\theta, \quad \theta_{2}=\theta+\frac{\pi}{2}, \quad \theta_{3}=\theta+\pi, \quad \theta_{4}=\theta+\frac{3 \pi}{2}, \\
r_{1}=r_{3}=r, \quad r_{2}=r_{4}=\rho r .
\end{gathered}
$$

The set of equations (21) now reduces to the set (22) for the determination of $r$ and $\theta$,

$$
\begin{aligned}
\frac{d^{2} r}{d t^{2}}-r\left(\frac{d \theta}{d t}\right)^{2}+\frac{1}{r^{2}}\left\{\frac{2 M}{\left(1+\rho^{2}\right)^{\frac{3}{2}}}+\frac{m}{4}\right\} & =0, \\
r \frac{d^{2} \theta}{d t^{2}}+2 \frac{d r}{d t} \frac{d \theta}{d t} & =0 .
\end{aligned}
$$

These are the equations for elliptic motion. Let $N=$ mean angular motion, and $A=$ major semi-axis. We have the relation

$$
N^{2} A^{3}=\frac{2 M}{\left(1+\rho^{2}\right)^{\frac{3}{2}}}+\frac{m}{4} .
$$

This is the analogue of the relation $N^{2} A^{3}=m_{1}+m_{2}$, which holds in the problem of two bodies.

YALE UNIVERSITY,

December, 1906. 